Revista de la Escuela de Ciencias de la Educación, año 14, nRo. 13, vol. 1, enero a junio de 2018. Páginas 149-165. ISSN 1851-6297 (DESDE DICIEMBRE DE 2006 A DICIEMBRE DE 2017). ISSN 2362-3349 (EN LINEA). VIDEOJUEGOS Y VIOLENCIA: Una ReVisión de la línea de inVestigación de los efectos. Santiago García Cernaz.

\title{
VIDEOJUEGOS Y VIOLENCIA: UNA REVISIÓN DE LA LÍNEA DE INVESTIGACIÓN DE LOS EFECTOS
}

\author{
Por Santiago García Cernaz* \\ Universidad Nacional de La Plata - Conicet, Argentina \\ santiagogarcia982@gmail.com
}

Recibido: 02/10/2017 Aceptado: 05/01/2017

\section{Resumen}

Los videojuegos son una actividad lúdica que tiene un lugar de privilegio en la vida cotidiana de las niñas, niños y jóvenes de las sociedades contemporáneas. Desde que hicieron su aparición hace algunas décadas, los contenidos violentos, presentes en un gran número de ellos, han despertado profundas controversias. Así, múltiples estudios se han llevado a cabo con el objetivo de determinar cuáles son los efectos de estos contenidos en los niños y jóvenes, principales destinatarios de esta propuesta lúdica. El presente artículo tiene como objetivo hacer una revisión de los principales desarrollos teóricos e investigaciones empíricas en psicología que se abocaron al estudio de estos efectos, enmarcándolos en el debate social más amplio en el que han tenido lugar. Se advierte la presencia de un sesgo de publicación y una falta de consenso acerca de la validez de las medidas de agresividad utilizadas. Asimismo, se ofrece una mirada alternativa para problematizar este enfoque.

Palabras clave: Videojuegos - Violencia - Agresividad - Efectos - Controversia.

\section{Abstract}

Video games are a playful activity that has a privilege place in the daily life of children and young people of contemporary societies. Since their appearance a few decades ago, violent content, present in a large number of them,

* Es Psicólogo por la Universidad Nacional del Comahue - UNCo y Doctorando del Doctorado en Psicología de la Universidad Nacional de La Plata. Actualmente se encuentra realizando una Beca Doctoral del Consejo Nacional de Investigaciones Científicas y Técnicas (CONICET). 
Revista de la Escuela de Ciencias de la Educación, año 14, nRo. 13, vol. 1, enero a junio de 2018. Páginas 149-165. ISSN 1851-6297 (DESDE DICIEMBRE DE 2006 A DICIEMBRE DE 2017). ISSN 2362-3349 (EN LINEA). VIDEOJUEGOS Y VIOLENCIA: Una revisión de la línea de investigación de los efectos. Santiago Garcia Cernaz.

has aroused deep controversy. Thus, multiple studies have been carried out with the objective of determining what are the effects of these contents on children and young people, main recipients of this contemporary playful proposal. This article aims to review the main theoretical basis and empirical research in psychology that focused on the study of these effects, framing them in the broader social debate in which they have taken place. It warns about the presence of a publication bias and a lack of consensus about the validity of the measures of aggressiveness used. Also, an alternative view is offered to problematize this approach.

Keywords: Video games - Violence - Aggressiveness - Effects - Controversy.

\section{Introducción}

Los videojuegos son una actividad lúdica que tiene un lugar de privilegio en la vida cotidiana de las niñas, niños y jóvenes de las sociedades contemporáneas. Desde Computer Space, que fue el primer videojuego comercializado en 1972, hasta los sofisticados videojuegos que actualmente están disponibles en el mercado tales como Grand Theft Auto 5 o FIFA 2016, han tenido lugar toda una serie de propuestas lúdicas mediadas por el video.

Actualmente, hay una amplia variedad de soportes para jugar videojuegos: máquinas arcade y simuladores, computadoras y notebooks, dispositivos móviles como tablets y teléfonos celulares, consolas domésticas y portátiles. Asimismo, existe una inacabable gama de géneros de videojuegos que día a día continúa ampliándose: simuladores de construcción, videojuegos de carreras, de luchas, de disparos, de guerra, de estrategias, de deportes, entre otros.

Ahora bien, los videojuegos han despertado toda una serie de controversias y posiciones encontradas en la sociedad desde que fueron lanzados al mercado. A medida que la oferta crecía y el número de jugadores iba en aumento, comenzó a gestarse también una representación negativa de los videojuegos con base en el supuesto de que son causantes de un amplio espectro de efectos indeseables: violencia, adicción, aislamiento social, bajo rendimiento académico, sedentarismo, deterioro de la imaginación, cefaleas, obesidad, entre otros (Tejeiro y Pelegrina, 2008; Jones, Scholes, Johnson, Katsikitis y Carras, 2014; Levis, 1997). Si bien existen amplios desarrollos que intentan recuperar su potencial educativo y motivacional, incluso algunos académicos los han considerado como una posible herramienta que transforme las condiciones educativas actuales y posibilite otros modos de aprendizaje más lúdicos y vinculados a los intereses de las nuevas generaciones (Buckingham, 2008), no es el caso de los videojuegos con contenidos más controversiales como el que aquí nos atañe: la violencia. 
Revista de la Escuela de Ciencias de la Educación, año 14, nRo. 13, vol. 1, enero a junio de 2018. Páginas 149-165. ISSN 1851-6297 (DESDE DICIEMBRE DE 2006 A DICIEMBRE DE 2017). ISSN 2362-3349 (EN LINEA). VIDEOJUEGOS Y VIOLENCIA: Una Revisión de la linea de investigación de los efectos. Santiago Garcia Cernaz.

\section{Una cronología del debate}

El tema de la violencia en los videojuegos ha estado presente en la literatura científica desde que fueron lanzados al mercado, siendo uno de los principales tópicos de investigación y debate (Estallo, 1995; Levis, 1997). Videojuegos arcade como Death Race de 1976, que permitía a los jugadores atropellar "gremlins" conduciendo un auto y obteniendo puntos con cada muerte, rápidamente dispararon controversias en el público.

En la década de 1980, sendos estudios se llevaron a cabo, abordando como objeto de análisis diversos títulos de gran popularidad en aquel entonces: Missile Command (que consistía en disparar contra misiles que caen del cielo), el archiconocido Pac-Man (que implica un personaje caricaturesco que come pelotitas en un laberinto mientras escapa de fantasmas), Berzerk (en donde un personaje bi-dimensional dispara a robots que lo persiguen), Zaxxon (un combate de naves aéreas) y Centipede (en el que el jugador defiende una parcela con hongos de insectos y arañas invasoras; v.g. Graybill, Strawniak, Hunter \& O'Leary, 1987; Anderson y Ford, 1986).

Ya en la década de 1990, los avances tecnológicos permitieron mayor complejidad en los juegos y mayor realismo en los gráficos: Street Fighter, Mortal Kombat (ambos juegos de peleas en tercera persona entre dos contrincantes) y Doom (uno de los primeros videojuegos de disparos en primera persona). En su lanzamiento para Nintendo, Mortal Kombat fue censurado: le quitaron la copiosa sangre y las brutales "fatalities" (1) dado que las consideraron como excesivamente violentas. Por el contrario, la consola Sega, optó por venderlo tal cual fue diseñado y el éxito en las ventas fue mucho mayor. Mortal Kombat es uno de los videojuegos que más controversias ha desatado por el nivel de violencia explícita que exhibe, llegando a la prohibición de su venta en algunos países.

De cara a los estallidos de violencia juvenil y tiroteos en escuelas e institutos que tuvieron lugar en los ' 90 (Ferguson, 2013), hubo quienes sostuvieron que los videojuegos estaban entrenando a los jóvenes para ser asesinos (Grossman, 1999). A pesar de estas declaraciones, los resultados de las investigaciones realizadas en los ' 80 y '90 no eran concluyentes (v.g. Cooper y Mackie, 1986; Dominick, 1984; Scott, 1995; Van Schie y Wiegman, 1997).

El punto de inflexión fue la tragedia del instituto Columbine (Wolf y Perron, 2005; Tejeiro y Pelegrina, 2008), en donde se reportó masivamente que los perpetradores del tiroteo eran acérrimos jugadores de Doom, "consolidando en la mente del público que los tiroteos escolares y los videojuegos violentos estaban invariablemente asociados" (Ferguson, 2013; p. 58. Traducido por quien suscribe).

La llegada del nuevo milenio trajo consigo también cambios notables en las tecnologías para el diseño de videojuegos, que posibilitaron el lanzamiento de nuevos títulos con mayores grados de realismo en los gráficos: juegos de 
Revista de la Escuela de Ciencias de la Educación, año 14, nRo. 13, vol. 1, enero a junio de 2018. Páginas 149-165. ISSN 1851-6297 (DESDE DICIEMBRE DE 2006 A DICIEMBRE DE 2017). ISSN 2362-3349 (EN LINEA). VIDEOJUEGOS Y VIOLENCIA: Una revisión de la línea de investigación de los efectos. Santiago Garcia Cernaz.

guerra como Medal of Honor o Call Of Duty, de acción y aventuras como Assassin's Creed o God Of Wary de disparos en primera persona como Counter Strike o Halo. También Grand Theft Auto, de acción/aventuras estilo sandbox, es un título que desde 1996 generaba controversias por tratarse de un juego cuya acción principal implica robar autos y atropellar peatones. Con la llegada de nuevos motores gráficos sus escenarios comenzaron a ser en 3-D, con el manejo del personaje desde una perspectiva de tercera persona y con mayores grados de realismo en las expresiones de violencia. Con Mortal Kombat ocurrieron procesos similares. Estas nuevas posibilidades reavivaron las posiciones más críticas respecto de los videojuegos.

Según Ferguson (2013), en este contexto de presión social para obtener respuestas de la ciencia frente a esta controversia, se observó un cambio sustancioso en las posiciones de los investigadores: a pesar de que no había resultados concluyentes, comenzaron a realizar declaraciones con un mayor grado de certidumbre acerca de los efectos de los videojuegos violentos en la conducta de los jugadores. Se comenzó a sostener que:

- Los efectos hallados eran similares en magnitud a aquellos encontrados en la correlación entre fumar cigarrillos y contraer cáncer de pulmón u otros efectos médicos importantes (Huesman, 2007).

- La violencia en los videojuegos y en otros medios podía explicar más del $30 \%$ de la violencia social (Strasburger, 2007).

- Los efectos de los videojuegos violentos eran comparables en magnitud a otros importantes factores de riesgo criminológicos (Anderson, 2004).

- Las inconsistencias y desacuerdos en el campo de investigación estaban virtualmente evaporadas. La Academia Americana de Pediatría (AAP), declaró en 2001 que "más de 3500 investigaciones han examinado la asociación entre la violencia en los medios y la conducta violenta; todos menos 18 han demostrado una relación positiva" (2001, citado en Kutner \& Olson, 2008. Traducido por quien suscribe). También la Asociación Americana de Psicología (APA) publicó en 2005 una resolución que sostenía que

un análisis exhaustivo de las investigaciones acerca de videojuegos interactivos violentos sugieren que tal exposición: a) aumenta los comportamientos agresivos b) aumenta las ideas agresivas c) aumenta los sentimientos de enojo d) disminuye comportamientos serviciales y, e) aumenta la excitación [arousal] fisiológica [...] Las características de la violencia en los videojuegos interactivos parecen tener efectos perjudiciales similares a la visualización de violencia en la televisión; sin embargo, basados en la teoría del aprendizaje (Bandura, 1977; Berkowitz), la práctica, la repetición, y las recompensas por actos de violencia pueden ser más propicios para aumentar los comportamientos agresivos en los niños y los jóvenes que mirar pasivamente violencia en la TV y en el cine (Traducido por quien suscribe. APA, 2005). 
Revista de la Escuela de Ciencias de la Educación, año 14, nRo. 13, vol. 1, enero a junio de 2018. Páginas 149-165. ISSN 1851-6297 (DESDE DICIEMBRE DE 2006 A DICIEMBRE DE 2017). ISSN 2362-3349 (EN LINEA). VIDEOJUEGOS Y VIOLENCIA: Una revisión de la línea de investigación de los efectos. Santiago García Cernaz.

En 2013, un grupo de 230 investigadores, criminólogos y psicólogos escribieron una carta abierta a la APA(Consortium of Scholars, 2013) solicitándole que retiren la resolución de 2005 dado que no había un consenso en la comunidad científica acerca de la validez de la relación causal que expresaron. En 2015 la APA publicó una resolución que modifica a la de 2005 en donde modera algunas afirmaciones, aunque conserva los aspectos centrales referentes a la importancia del efecto que tiene el uso de videojuegos en la agresividad de los jugadores (APA, 2015).

\section{Principales aproximaciones teóricas}

El mecanismo más frecuentemente citado según el cual el uso de videojuegos puede conducir al comportamiento agresivo es la teoría del aprendizaje social (Sherry, 2001). Dicha teoría plantea que el comportamiento es aprendido a través de la observación e imitación del comportamiento de los otros, quienes constituyen genuinos modelos sociales (Bandura, 1994). Aquellos comportamientos que son recompensados tienden a volverse más permanentes en el repertorio conductual del aprendiz que los que son castigados. Los autores que proponen la teoría del aprendizaje social para comprender la relación de los jugadores con los videojuegos argumentan que éstos deben tener un poderoso efecto debido a los altos niveles de atención que conllevan y por la identificación activa de los jugadores con los personajes de la pantalla (v.g. Brusa, 1988). Además, sostienen, al ser recompensados directamente por ejecutar acciones violentas podrían transferir esta agresividad aprendida hacia el mundo exterior.

Un segundo modelo afirma que los videojuegos tienen un efecto en los jugadores por medio de un mecanismo de priming o activación de redes neo-asociativas (v.g. Anderson \& Ford, 1986). Se basa en los estudios de la influencia de los medios de comunicación en los individuos de Berkowitz (Berkowitz \& Rogers, 1986) quien postuló la teoría del efecto de priming. Según dicho autor, los contenidos violentos de los medios conducen a la agresividad o a la hostilidad debido a la activación de nodos informacionales semánticamente asociados por medio de redes. En el caso de los videojuegos, la teoría del efecto de priming sugiere que la exposición a los contenidos violentos activará una serie de nodos asociados con la violencia y la agresividad. La activación de estos nodos posibilita la transferencia de pensamientos agresivos a la acción.

Otra aproximación teórica de los efectos de los videojuegos está constituida por el modelo general del arousal o alerta (v.g. Calvert \& Tan, 1994). El modelo elemental del arousal de Tannenbaum y Zillmann (1975) propone que el arousal es un estado inespecífico de activación. Este estado puede variar desde un nivel basal de atención óptimo para la acción intencional hasta un estado de sobre-activación, como en el caso de emociones intensas o de es- 
Revista de la Escuela de Ciencias de la Educación, año 14, nRo. 13, vol. 1, enero a junio de 2018. Páginas 149-165. ISSN 1851-6297 (DESDE DICIEMBRE DE 2006 A DICIEMBRE DE 2017). ISSN 2362-3349 (EN LiNEA). VIDEOJUEGOS Y VIOLENCIA: Una revisión de la línea de investigación de los efectos. Santiago Garcia Cernaz.

tados de alerta, como la lucha o la huida. Los medios de comunicación, como las películas o la televisión, tienden a intensificar el actual estado de excitación $y$, por ende, a potenciar las reacciones del individuo. Desde este punto de vista, los videojuegos altamente violentos proveen la activación que es necesaria para intensificar las respuestas agresivas.

Una cuarta teoría propone que el uso de videojuegos violentos puede tener un resultado benéfico para los jugadores: se trata del efecto de catarsis. Según estos autores, las acciones violentas realizadas en los videojuegos son un modo seguro de descarga de los pensamientos y sentimiento agresivos de los jugadores (v.g. Calvert y Tan, 1994). Esta teoría fue originalmente postulada por Feshbach (1995), quien planteaba que los individuos pueden descargar sus sentimientos agresivos mirando una representación ficticia de violencia en los medios. Kestenbaum y Weinstein (1985), afirman que la naturaleza interactiva de los videojuegos permite a los jugadores exteriorizar una agresividad que no está permitida en el mundo real.

Finalmente, una de las más recientes aproximaciones teóricas que se han propuesto es el denominado Modelo General de Afectividad y Agresividad (General Affective Agression Model - GAAM) (v.g. Carnagey y Anderson, 2003; Anderson y Dill, 2000; Anderson et al., 2010). El modelo constituye un enfoque comprensivo que abreva de los aportes de numerosos enfoques acerca de la agresividad e intenta articularlos en un marco integrador. El modelo describe al comportamiento agresivo como un proceso multi-etapa que inicia con un input o estímulo y concluye con un output o respuesta, mediado por el estado interno del individuo y la evaluación de la situación que éste realiza. Los estímulos pueden ser tanto situacionales (v.g. amenaza) como de personalidad (v.g. personalidad agresiva) y ambos influyen en el estado interno del individuo, el cual está constituido por tres niveles: cogniciones, emociones y nivel de arousal. El estado interno, a su vez, condiciona la evaluación que el individuo realice de la situación (v.g. amenaza) llevándolo a una respuesta automática o controlada. Por ejemplo, según el modelo, un individuo cuyos script o guiones de acción (nivel cognitivo) implican una respuesta de ataque frente a una situación de amenaza, tenderá a responder automáticamente con el ataque y más aún si su nivel de arousal en ese momento es elevado y experimenta un afecto de hostilidad. Por el contrario, un individuo cuyos scripts implican la huida frente a una amenaza, emparejado con el afecto del miedo, tenderá a responder automáticamente con la evitación del enfrentamiento. Teóricamente, cualquiera de las tres variables del estado interno del individuo, i.e., las cogniciones, las emociones y el arousal, puede influir a las otras dos y desencadenar respuestas automáticas o controladas. Éstas últimas implican mayores recursos cognitivos para inhibir las respuestas automáticas, que son activadas de modo inmediato. El modelo predice que la violencia presente en los videojuegos conduce a un aumento de la agresividad de los jugadores, 
Revista de la Escuela de Ciencias de la Educación, año 14, nRo. 13, vol. 1, enero a junio de 2018. Páginas 149-165. ISSN 1851-6297 (DESDE DICIEMBRE DE 2006 A DICIEMBRE DE 2017). ISSN 2362-3349 (EN LINEA). VIDEOJUEGOS Y VIOLENCIA: Una Revisión de la linea de investigación de los efectos. Santiago Garcia Cernaz.

tanto a corto como a largo plazo, dado que esta exposición activa pensamientos agresivos e incrementa las emociones hostiles y el nivel de arousal. Según Anderson y Dill (2000):

Cada vez que las personas juegan a videojuegos violentos, repiten scripts agresivos que enseñan y refuerzan la vigilancia hacia los enemigos (i.e. sesgos de percepción hostiles), acciones agresivas contra los otros, expectativas de que los otros se comporten agresivamente, actitudes positivas frente al uso de la violencia y creencias de que las soluciones agresivas son efectivas y apropiadas. Más aún, es muy probable que la exposición repetida a escenas gráficas de violencia sea desensibilizante. [...] Los jugadores de videojuegos a largo plazo pueden volverse más agresivos en sus perspectivas, sesgos de percepción, actitudes, creencias y comportamientos de lo que eran antes de la exposición reiterada o de lo que habrían sido sin esa exposición. (p. 774. Traducido por quien suscribe).

Según el modelo, la exposición reiterada a videojuegos violentos, y a la violencia en otros medios también, puede, a largo plazo, transformar la personalidad del individuo haciéndolo más agresivo.

\section{Principales resultados de los estudios empíricos}

Tres grupos de investigadores distintos han llevado a cabo meta-análisis de los estudios que abordan estos efectos (Anderson et al., 2010; Ferguson, 2007; Ferguson \& Kilburn, 2009; Sherry, 2001). Aunque las magnitudes del efecto reportadas por éstos son similares $(r=0.19, r=0.15, r=0.16$ y $r=$ 0.16 , respectivamente), las interpretaciones son muy disímiles. Según Anderson et al. (2010), esta magnitud del efecto debería ser más alta puesto que se encuentra disminuida por las fallas metodológicas de numerosos estudios, entre las cuales se mencionan las medidas de agresividad poco rigurosas. Este punto lo desarrollaremos más adelante. Por su parte, tanto Sherry (2001) como Ferguson y Kilburn (2009) y Ferguson (2007) sostienen que esta cifra puede estar incluso "inflada" por distintos factores.

Ferguson y Kilburn (2009) y Ferguson (2007) en su meta-análisis han hallado que existe un sesgo de publicación. También denominado como "el problema del archivo encajonado" (Kutner \& Olson, 2008), este sesgo ocurre cuando los artículos con resultados positivos, es decir, estadísticamente significativos, son seleccionados para su publicación en una proporción mayor que los artículos cuyos resultados son negativos. Por ende, la literatura disponible en publicaciones arbitradas puede proveer una muestra sesgada de todos los estudios que realmente se llevaron a cabo, exhibiendo más resultados positivos de los que en verdad existen. Este sesgo puede afectar los meta-análisis, dado que muchos de los estudios no-publicados son difíciles de localizar y por ende no son incluidos. Goldstein sostiene: 
Revista de la Escuela de Ciencias de la Educación, año 14, nRo. 13, vol. 1, enero a junio de 2018. Páginas 149-165. ISSN 1851-6297 (DESDE DICIEMBRE DE 2006 A DICIEMBRE DE 2017). ISSN 2362-3349 (EN LINEA). VIDEOJUEGOS Y VIOLENCIA: Una revisión de la línea de investigación de los efectos. Santiago Garcia Cernaz.

La investigación publicada en revistas académicas no representa toda la investigación en juegos electrónicos. Los estudios que fracasan en hallar resultados estadísticamente significativos tienen menos probabilidades de ser aceptados para su publicación. Entonces los registros publicados son una desconocida fracción de toda la investigación, y tiende a estar constituida por aquellos estudios con resultados estadísticamente significativos. Esto se conoce como el "problema del archivo encajonado" porque los estudios que no hayan ningún efecto de los videojuegos permanece sin publicar, encajonado en los archivos del investigador. (Goldstein, citado en Kutner \& Olson, 2008, p. 22).

Este sesgo, muy probablemente, puede ocurrir en dos niveles. En primer lugar, los editores de las revistas científicas pueden preferir los estudios con hallazgos "positivos" para su publicación dado que creen que serán más interesantes para sus lectores. Por consiguiente, los manuscritos con hallazgos "negativos" son rechazados más frecuentemente. En segundo lugar, los autores mismos pueden eliminar investigaciones que no confirman sus hipótesis, ya sea suprimiendo un estudio completo con resultados "negativos" o volviendo a ejecutar el análisis estadístico de los datos usando múltiples variaciones hasta que produzcan los resultados deseados. Ferguson (2007) argumenta que "esto no implica necesariamente una conducta intencionalmente no ética, como investigadores pueden interpretar los resultados iniciales como una "equivocación" o como un "error" inicial de su parte que, una vez corregido, produce los resultados esperados" (p. 473. Traducido por quien suscribe). Ferguson y Kilburn (2009) realizaron un análisis estadístico que permite conocer cuál sería la magnitud del efecto si este sesgo de publicación hallado fuese corregido bajaría a $r=.08$.

Además, tanto Sherry (2001) como Ferguson y Kilburn (2009) han encontrado que la magnitud del efecto puede estar "inflada" por la presencia de variables moderadoras. Sherry (2001) halló que el tiempo de juego, la edad de los sujetos y los distintos tipos de violencia que poseen, tienen un influjo poderoso en los efectos hallados.

Incluso tomando los valores del efecto, hallados en los meta-análisis mencionados, como carentes de sesgos o variables moderadoras, constituyen unos efectos que, convertidos a la $d$ de Cohen, puede ser categorizados como pequeños ( $d=.3$ ) (Cf. Coe y Merino Soto, 2003). No proporcionan una base sólida para situarla en el mismo nivel de otros factores de riesgo criminológicos importantes como el abuso de sustancias o la presencia de padres abusivos tal como sostienen, por ejemplo, Anderson et al (2010). Tampoco justifica las generalizadas analogías que se han establecido con la relación entre fumar tabaco y contraer cáncer de pulmón. A propósito de ello, Ferguson y Kilburn (2009) sostienen: 
Revista de la Escuela de Ciencias de la Educación, año 14, nRo. 13, vol. 1, enero a junio de 2018. Páginas 149-165. ISSN 1851-6297 (DESDE DICIEMBRE DE 2006 A DICIEMBRE DE 2017). ISSN 2362-3349 (EN LINEA). VIDEOJUEGOS Y VIOLENCIA: Una revisión de la línea de investigación de los efectos. Santiago García Cernaz.

Este trabajo pone en cuestión la significancia de la exposición a la violencia en los medios como un problema de salud pública. Según las más liberales estimaciones disponibles [...], los efectos observados en fumar y tener cáncer de pulmón son al menos 8 veces más fuertes que [los efectos de] la exposición a la violencia en los medios. Usando las cifras más conservadoras [...] es un número cercano a 135 veces más fuerte. Para poner esto en el contexto del comportamiento violento, la magnitud del efecto de otras variables relacionadas con la violencia abarcan la genética $(r=.75)$, auto-control personal y oportunidad criminal $(r=.58)$, pobreza $(r=.25)$, y la exposición a abuso físico en la infancia $(r=.25)$. (p. 762. Traducido por quien suscribe).

Guy Cumberbatch, psicólogo director del Communications Research Group en Gran Bretaña, ha estudiado los efectos de los medios masivos en la conducta violenta por varias décadas. Resume:

El verdadero enigma es que cualquiera que mire las evidencias de las investigaciones en este campo puede sacar cualquier conclusión acerca del patrón, por no decir que se argumenta con tal confianza y hasta pasión que está demostrado el daño de la violencia en la televisión, en el cine y en los videojuegos. Si bien las pruebas de significancia estadística son una herramienta vital de las ciencias sociales, parece que han sido más a menudo usadas en este campo como instrumentos de tortura de los datos hasta que confiesen algo que pueda justificar su publicación en una revista científica. [...] La violencia en los medios ha sido sometida a una mentalidad de linchamiento en masa con casi ninguna evidencia usada para probar su culpabilidad. (Cumberbatch, citado en Kutner y Olson, 2008, p. 9).

Ferguson (2015) sugiere que la defensa de la tesis de que existe una fuerte relación, incluso causal, entre el uso de videojuegos violentos y la conducta agresiva o violenta de los jugadores está influenciada por narrativas sociales sobre los efectos de los medios que hacen eco del pánico moral del que otrora fue objeto el cine, los comics, el rock y la televisión (Cf. también Kutner y Olson, 2008).

Asimismo, este tipo de enfoques, muy frecuentemente, utilizan definiciones poco precisas de "agresividad" y "violencia" (v.g. Sherry, 2001; Kutner y Olson, 2008). A menudo se utilizan como sinónimos intercambiables, dando por supuesto que uno conduce al otro. La violencia podemos definirla como una acción intencionada de herir física o psicológicamente a otro o de arrebatarle un derecho que le es propio. La agresividad, por el contrario, no es tan sencilla de definir. Una definición a menudo utilizada por psicólogos afirma que es "un comportamiento conducente a la auto-afirmación; puede surgir de impulsos innatos y/o como respuesta a la frustración, y puede manifestarse por conductas destructivas o de ataque, por hostilidad u obstruccionismo, o 
Revista de la Escuela de Ciencias de la Educación, año 14, nRo. 13, vol. 1, enero a junio de 2018. Páginas 149-165. ISSN 1851-6297 (DESDE DICIEMBRE DE 2006 A DICIEMBRE DE 2017). ISSN 2362-3349 (EN LINEA). VIDEOJUEGOS Y VIOLENCIA: Una revisión de la línea de investigación de los efectos. Santiago Garcia Cernaz.

por impulsos auto-expresivos de dominio" (citado en Kutner y Olson, 2008, p. 15. Traducido por quien suscribe).

De este modo, hay comportamientos que bien pueden ser agresivos pero no violentos, como por ejemplo, los que tienen lugar a menudo en el juego infantil. Es algo muy usual entre los niños el jugar a "luchitas" o a derribarse mutuamente, pero sin la intención de lastimar al otro seriamente. La agresividad está presente en muchas formas de juego y en distintos deportes, siendo un componente clave de la auto-afirmación que se necesita para enfrentarse al contrincante, aunque sin implicar por ello una conducta violenta que tenga por objetivo herir al otro.

Esta distinción muchas veces es pasada por alto por los investigadores, la cual está íntimamente relacionada con la frecuente falta de validez de las medidas de agresividad que se utilizan en los laboratorios de psicología para realizar estos estudios (Anderson et al., 2010; Sherry, 2001; Ferguson \& Kilburn, 2009; Kutner y Olson, 2008). Uno de los tests más usados para medir la agresividad es el Taylor Competitive Reaction Time Test (TCRTT), que ha sido ya criticado por su generalizado uso de modo no estandarizado (Ferguson, 2015; Kutner y Olson, 2008). Brevemente, en el TCRTT el sujeto cree que el test consiste en un juego de tiempo de reacción contra otra persona. Cuando pierde, al sujeto se le lanza un estallido de ruido blanco que cree que su oponente ha disparado contra él. Cuando gana, puede lanzar un estallido similar contra su oponente. En realidad, no hay ningún oponente: las victorias y las derrotas de los participantes están programadas previamente en una computadora.

En el TCRTT la agresividad está definida operacionalmente como la medida de la intensidad y la duración de los estallidos de ruido blanco que el participante decide disparar contra su supuesto oponente. Se computan tanto el número de victorias y derrotas del participante como la intensidad y la duración de los estallidos que dispara, aunque cada una de estas cuatro dimensiones son interpretadas y analizadas de modo diferente por los distintos investigadores e incluso por el mismo investigador en estudios consecutivos (v.g. Anderson y Dill, 2000; Anderson y Murphy, 2003; Anderson et al., 2004; Carnagey y Anderson, 2005; Anderson y Carnagey, 2009). Por consiguiente, "existe la posibilidad de que diferentes índices puedan mostrar que los videojuegos aumentan, disminuyen o no tienen efecto en la agresión del jugador para una misma muestra de participantes" (Ferguson, 2013, p. 64. Traducido por quien suscribe).

Otro problema de la utilización del TRCTT es que nunca fue validado externamente: no hay estudios que vinculen estas medidas con actividades violentas criminales reales (Ferguson, 2007). Ferguson argumenta: 
Revista de la Escuela de Ciencias de la Educación, año 14, nRo. 13, vol. 1, enero a junio de 2018. Páginas 149-165. ISSN 1851-6297 (DESDE DICIEMBRE DE 2006 A DICIEMBRE DE 2017). ISSN 2362-3349 (EN LINEA). VIDEOJUEGOS Y VIOLENCIA: Una revisión de la línea de investigación de los efectos. Santiago García Cernaz.

La mayoría de la gente externa a la investigación asocia la agresión al intento de herir físicamente a alguien. Si el estallido de sonido se ajusta a algo como eso es muy discutible. Mi parecer es que en realidad es una mejor medida de competitividad, lo cual está justo en su nombre: Taylor Competitive Reaction Time Test. En mi opinión, está muy lejos de golpear a alguien hasta la muerte, o siquiera azotar o abofetear a alguien, porque no es así de aversivo. (citado en Kutner y Olson, 2008, p. 16. Traducido por quien suscribe).

Otros métodos de medir la agresividad aplicados en diseños de laboratorio incluyen medidas fisiológicas de arousal o excitación relativamente inocuas tales como ondas cerebrales (Bartholow, Bushman y Sestir, 2006), ritmo cardíaco (Fleming y Rickwood, 2001), pensamientos agresivos (Anderson y Dill, 2000) u otras medidas como interpretar las acciones de un personaje en una historia (Bushman y Anderson, 2002) y sentenciar criminales en un escenario análogo (Deselms y Altman, 2003). En otro estudio con población infantil, luego de haber jugado un videojuego violento se les solicitó a los participantes que completen un inventario de palabras incompletas tales como "explo_ar", de tal modo que aquellos niños que completaban con " $r$ " denotaban menos agresividad que aquellos que completaban con "t" (Ferguson, 2013). Ninguna de estas medidas de "agresividad" fue asociada con conductas violentas reales o conductas criminales. "Esta distinción es a veces ignorada en el público general (aunque también por algunos estudiosos [...]) y puede a veces ser desatendida cuando la discusión se enfoca en la salud pública..." (Ferguson, 2013, p. 63. Traducido por quien suscribe).

\section{Reflexiones finales: ¿Efectos?}

La línea de investigación que aquí revisamos descansa, entonces, en el supuesto de que existe un efecto en los jugadores a causa de la exposición a los videojuegos. Coe y Merino Soto (2003) señalan que a menudo se utiliza de modo ambiguo el término "efecto" cuando los estudios, tanto correlacionales como experimentales, ofrecen el valor de la magnitud del efecto de sus hallazgos:

la palabra efecto tiene una inherente implicación de causalidad: hablar de "el efecto de A sobre B" sugiere una relación causal más que solo una asociación. Desgraciadamente, sin embargo, la palabra efecto es frecuentemente utilizada cuando no se hace una afirmación causal explícita, sino que su implicación es algunas veces permitida que salga a flote dentro y fuera del significado, aprovechando la ventaja de la ambigüedad de sugerir un vínculo causal donde realmente nada se justifica para ello. (p. 170).

Es notorio el hecho de que a pesar de que se han dedicado enormes esfuerzos por cuantificar este efecto de los videojuegos violentos, no se han ha- 
Revista de la Escuela de Ciencias de la Educación, año 14, nRo. 13, vol. 1, enero a junio de 2018. Páginas 149-165. ISSN 1851-6297 (DESDE DICIEMBRE DE 2006 A DICIEMBRE DE 2017). ISSN 2362-3349 (EN LiNEA). VIDEOJUEGOS Y VIOLENCIA: Una revisión de la línea de investigación de los efectos. Santiago Garcia Cernaz.

llado aún evidencias de peso. Muy probablemente se deba a que el problema no se está planteando del modo más adecuado. Sostener que los jugadores son objeto de un efecto del videojuego que puede cuantificarse, los posiciona como una masa homogénea de receptores pasivos que son víctimas de la influencia de un poderoso estímulo que modifica su conducta de manera directa. Este enfoque, que también podemos observar en estudios realizados con otros medios como la televisión o el cine, hace eco de una visión que hace tiempo ha caído en descrédito: la teoría de la aguja hipodérmica (Lasswell, 1948).

Esta teoría, desarrollada en los albores de la cultura de masas y en el contexto de los grandes programas de propaganda política del período de entre guerras, supone un modelo del comportamiento humano que retoma los supuestos del conductismo clásico. Según este punto de vista, los medios de comunicación son omnipresentes y la audiencia es homogénea y manipulable: el estímulo mediático impacta directamente sobre el individuo y gatilla una respuesta inmediata, siguiendo una lógica lineal de causa-efecto. El individuo queda así reducido a una pasiva "víctima" de una poderosa, inmediata y directa influencia de los medios. Si bien hace tiempo que ha caído en descrédito (Shaw, 1979) y ha sido superada por teorías más complejas en el campo de la comunicación, constituye una visión que no se ha abandonado del todo. Inclusive las teorías que dan un giro "cognitivo" al enfoque de los efectos, como el GAAM desarrollado más arriba, y que consideran instancias cognitivas mediadores entre el estímulo y la respuesta conductual, aun así conciben la asociación entre input y output como un proceso poderosamente determinado por el conjunto de los refuerzos y castigos de las contingencias ambientales.

En numerosos estudios que han tenido como objeto la relación de los niños con la televisión pueden observarse elementos de esta perspectiva (Dotro, 1999). Estos estudios afirman que la transmisión televisiva de un determinado tipo de valores, estereotipos sociales, ideas, sentimientos, normas morales, etc., inducen a los niños de modo directo a pensar, sentir y actuar de una determinada forma (Leal, 2002).

Quienes adscriben a este posicionamiento consideran que la televisión es un medio pasivo, anti-intelectual que anima a los niños/as a "vegetar", que los/ as pervierte y hasta les genera adicción (Greenfield, 1985; Propper, 2007). Al igual que con los estudios posteriores que se abocaron al análisis de los videojuegos, aducen que lo más preocupante es la violencia que transmiten los programas televisivos, puesto que incide en la mente y el comportamiento agresivo de los niños y niñas, con el consecuente peligro social que esto conlleva.

En este enfoque subyace la idea del niño gestada en la modernidad: la de un niño pasivo, permeable, caracterizado por el desvalimiento, la desprotección, la indefensión, la fragilidad, la ductilidad y la maleabilidad. Según 
Revista de la Escuela de Ciencias de la Educación, año 14, nRo. 13, vol. 1, enero a junio de 2018. Páginas 149-165. ISSN 1851-6297 (DESDE DICIEMBRE DE 2006 A DICIEMBRE DE 2017). ISSN 2362-3349 (EN LINEA). VIDEOJUEGOS Y VIOLENCIA: Una Revisión de la linea de investigación de los efectos. Santiago Garcia Cernaz.

Pindado (1997) supone una concepción de la mente como un "vacuum", un recipiente vacío, de modo que cada mensaje actúa como un estímulo-resorte que empuja al individuo a la acción. El niño, desde esta perspectiva, es naturalmente "sano" y por imitación y/o reacción de lo que ve en la pantalla se torna agresivo y violento. Asimismo, prevalece una visión más funcionalista del proceso de socialización, según la cual el agente socializador moldea la conducta de los niños de modo directo y unidireccional (Lezcano, 1998).

La pretensión de cuantificar un efecto de la exposición a un medio, supone, de antemano, cierta homogeneidad en la recepción del mismo, es decir, no atiende a la heterogeneidad de los modos singulares en que cada individuo interactúa con el medio y se apropia o re-construye internamente los mensajes que se transmiten. La utilización de la noción misma de exposición para denotar esta relación entre el medio y el individuo implica esta idea de pasividad. Supone, implícitamente, que hay una relación de causa-efecto entre el mensaje del medio y la conducta posterior del jugador, sin tomar en consideración otros factores que puedan intervenir en la relación entre el jugador y el contenido del videojuego. La conducta violenta es un problema complejo que no puede ser comprendido si se utiliza una lógica lineal de causas y efectos.

Kutner y Olson (2008) aseguran que las medidas de agresividad que usualmente se utilizan dentro de esta línea de investigación, y que hemos analizado anteriormente, no consideran las diferencias individuales entre los distintos jugadores en lo que respecta a, por ejemplo, personalidad o estilos de comportamiento. Unsworth, Devilly y Ward (2007) han encontrado que los jugadores pueden experimentar tanto aumentos, disminuciones o ausencia de cambios en su agresividad luego de jugar un videojuego violento, y que ello está fuertemente vinculado con los sentimientos de los jugadores en los momentos previos al uso del videojuego.

Kutner y Olson (2008) realizaron una investigación desde un enfoque "epidemiológico", que buscó identificar los diferentes patrones de uso de videojuegos en una amplia muestra de niños y jóvenes jugadores. Señalan que en niños y niñas con personalidad agresiva o que están atravesando eventos vitales estresantes hay una relación fuerte entre el uso muy frecuente de videojuegos violentos y un alto riesgo de bullying o peleas físicas. Los autores aseguran que es probable que los niños agresivos se sientan más atraídos por los videojuegos violentos o que los niños que estén bajo estrés, por problemas familiares o escolares, utilicen los videojuegos para manejar ese estrés.

Por consiguiente, una elevada frecuencia de uso de videojuegos con contenidos violentos y de modo solitario, más que ser causante de "trastornos", puede interpretarse como síntoma indicador de un padecimiento que está atravesando el niño, ya sea en su ambiente familiar o escolar, y que eventualmente exige intervención adulta. Reflexionar desde esta perspectiva, nos posibilita, como padres y educadores, posicionarnos desde un lugar de mayor 
Revista de la Escuela de Ciencias de la Educación, año 14, nRo. 13, vol. 1, enero a junio de 2018. Páginas 149-165. ISSN 1851-6297 (DESDE DICIEMBRE DE 2006 A DICIEMBRE DE 2017). ISSN 2362-3349 (EN LINEA). VIDEOJUEGOS Y VIOLENCIA: Una revisión de la línea de investigación de los efectos. Santiago Garcia Cernaz.

empatía con la realidad del niño. Para establecer un vínculo de genuino acompañamiento, será necesario no sólo observar cuáles son los contenidos de los juegos que los niños utilizan, sino en qué momentos los utilizan, con qué fines, si juegan solos o en compañía y, principalmente, posibilitar un espacio de escucha atenta de los sentimientos que les provocan y las ideas que construyen acerca de esos contenidos. De este modo, la intervención adulta dejaría de estar planteada desde un marco meramente prohibitivo o restrictivo, implícito en un enfoque de los efectos en el que, para evitar el supuesto daño, es necesaria la total sustracción del estímulo presuntamente pernicioso.

Creemos entonces, para concluir, que esta forma alternativa de enfocar el problema nos abre nuevos horizontes para comprender los complejos avatares que actualmente transitan los niños y jóvenes en su proceso de socialización, en donde las tecnologías de la información y la comunicación han llegado para quedarse.

\section{Notas bibliográficas}

(1) Ataque final de la pelea en la que el personaje realiza un poder sobrehumano o una secuencia de golpes que conllevan la mutilación y aniquilamiento del contrincante.

\section{Referencias bibliográficas}

- American Psychological Association (2005). Resolution on Violent Video Games and Interactive Media. Recuperada de https://www.apa.org/about/policy/interactive-media. pdf

- American Psychological Association (2015). Resolution on Violent Video Games. Recuperado de http://www.apa.org/about/policy/violent-video-games.aspx

- Anderson, C. A. \& Carnagey, N. L. (2009). Causal effects of violent sports video games on aggression: Is it competitiveness or violent content?. Journal of Experminetal Social Psychology, 43, pp. 731-739.

- Anderson C. A.; Carnagey, N. L.; Flanagan, M.; Benjamin, A J.; Eubanks, J. \& Valentine, J. C. (2004). Violent video games: Specific effects of violent content on aggressive thoughts and behavior. Advances in Experiment Social Psychology, 36, pp. 199-249.

- Anderson C. A. \& Dill, K. (2000). Video games and aggressive thoughts, feelings and behavior in the laboratory and in life. Journal of Personality and Social Psichology, 78, pp. 772-790.

- Anderson, C. A. \& Ford, C. M. (1986). Affect of the game player: short-term effects of highly and mildly aggressive video games. Personality and Social Psychology Bulletin, 12(4), pp. 390-392.

- $\quad$ Anderson C. A. \& Murphy, C R. (2003). Violent video games and aggressive behavior in young women. Aggressive Behavior, 29, pp. 423-229.

- Anderson, C. A.; Shibuya, A.; Ihori, N.; Swing, E. L.; Bushman, B. J.; Sakamoto, A.; Rothstein, H. R. \& Saleem, M. (2010). Violent video games effects on aggression, empathy, and prosocial behavior in Eastern and Wetern contries: a meta-analytic review. Psychological Bulletin, 136(2) pp. 151-173. doi 10.1037/a0018251 
Revista de la Escuela de Ciencias de la Educación, año 14, nRo. 13, vol. 1, enero a junio de 2018. Páginas 149-165. ISSN 1851-6297 (DESDE DICIEMBRE DE 2006 A DICIEMBRE DE 2017). ISSN 2362-3349 (EN LINEA). VIDEOJUEGOS Y VIOLENCIA: Una Revisión de la linea de investigación de los efectos. Santiago Garcia Cernaz.

- Bandura, A. (1994). The social cognitive theory of mass communication. En: J. Bryant y D. Zillmann (Eds.), Media effects: advances in theory and research, pp. 61-90. Hillsdale, NJ: Erlbaum.

- Bartholow, B.; Bushman, B. \& Sestir, M. (2006). Chronic violent video game exposure and desensitation to violence: brain potential data. Journal of Experimental Social Psychology, 42, pp. 532-539.

- $\quad$ Berkowitz, L. \& Rogers, K.H. (1986). A priming effect analysis of media influences. En J. Bryant y D. Zillmann (Eds.). Perspectives on media effects, pp. 57-81. Hillsdale, NJ: Erlbaum.

- Brusa, J.A. (1988). Effects of video game playing on children's social behavior (aggression, cooperation). Dissertation Abstracts International-B, 48(10), 3127. (University Microfilms No. 8800625).

- Buckingham, D. (2008). Más allá de la tecnología. Aprendizaje infantil en la era de la cultura digital. Buenos Aires: Ediciones Manantial.

- Bushman, B. y Anderson, C. (2002). Violent video games and hostile expectations: a test of the General Aggression Model. Personality and Social Psychology Bulletin, 28, pp. 1679-1686.

- Calvert, S. y Tan, S.L. (1994). Impact of virtual reality on young adults' physiological arousal and aggressive thoughts: interaction versus observation. Journal of Applied Developmental Psychology, 15, pp. 125-139.

- Carnagey, N. L. y Anderson, C. A. (2005). The effects of reward and punishment in violent video games on aggressive affect, cognition and behavior. Psychological Science, 16, pp. 882-889.

- Coe, R. y Merino Soto, C. (2003). Magnitud del efecto: una guía para investigadores y usuarios. Revista de Psicología - PUCP, 21(1), pp.145-177.

- Cooper, J. y Mackie, D. (1986). Video games and aggression in children. Journal of Applied Social Psychology, 16, pp. 726-744. doi 10.1111/j.1559-1816.1986.tb01755.x

- Consortium of Scholars (2013). Scholar's open statement to the APA task force on violent media. Recuperado de http://www.christopherjferguson.com/APA\%20Task\%20 Force\%20Comment1.pdf

- Deselms, J. \& Altman, J. (2003). Inmediate and prolonged effects of video game violence. Journal of Applied Social Psychology, 33, pp. 1553-1563.

- Feshbach, S. (1995). The drive-reducing function of fantasy behavior. Journal of Abnormal and Social Psychology, 50, pp.3-11.

- Dominick, J. (1984). Video games, television violence and aggression in teenagers. Journal of Communication, 34 (2), pp. 136-147. doi: 10111/j.1460-2466.1984.tb02165.x

- Dotro, M. V. (1999). Infancia y Televisión. Un debate sin tregua. Ensayos y Experiencias, 6(31).

- Estallo, J. A. (1995). Los videojuegos. Juicios y prejuicios. Barcelona: Planeta.

- Ferguson, C. J. (2007). Evidence for publication bias in video game violence effects literature: a meta-analytic review. Aggression and Violent Behavior, 12, pp.470-482. doi 10.1016/j.avb.2007.01.001

- Ferguson, C. J. (2013). Violent Video Games and the Supreme Court. Lessons for the Scientific Community in the Wake of Brown v. Entertainment Merchants Association. 
Revista de la Escuela de Ciencias de la Educación, año 14, nRo. 13, vol. 1, enero a junio de 2018. Páginas 149-165. ISSN 1851-6297 (DESDE DICIEMBRE DE 2006 A DICIEMBRE DE 2017). ISSN 2362-3349 (EN LINEA). VIDEOJUEGOS Y VIOLENCIA: Una revisión de la línea de investigación de los efectos. Santiago Garcia Cernaz.

American Psychologist, 68(2), pp.57-74.

- Ferguson, C. J. (2015). Does Media Violence Predict Societal Violence? It Depends on What You Look at and When. Journal of Communication, 65, pp.1-22.

- Ferguson, C. J. \& Kilburn, J. (2009). The public health risks of media violence: a meta-analytic review. Journal of Pediatrics, 154(5), pp.759-763. doi:10.1016/j. jpeds.2008.11.033

- Fleming, M. \& Rickwood, D. (2001). Effects of violent versus nonviolent video games on childrens' arousal, aggressive mood and positive mood. Journal of Applied Social Psychology, 31, pp.2047-2071.

- Graybill, D.; Strawniak, M.; Hunter, T. y O'Leary, M. (1987). Effects of playing versus observing violent versus nonviolent video games on children's aggression. Psychology: A Quarterly Journal of Human Behavior, 24, pp. 1-8.

- Greenfield, P. (1985). El niño y los medios de comunicación. Madrid: Morata.

- Grossman, D. (1999). On killing: The psychological cost of learning to kill in war and society. Boston, MA: Back Bay Books.

- Huesman, L.R. (2007). The impact of electronic media violence: scientific theory and research. Journal of Adolescent health, 41, S6-S13. doi 10.1016/j.jadohealth.2007.09.05

- Jones, C. M.; Scholes, L.; Johnson, D.; Katsikitis, M., \& Carras, M. C. (2014). Videogames: dispelling myths and tabloid headlines that videogames are bad. Proceedings of the $28^{\text {th }}$ International BCS Human Computer Interaction Conference (HCl 2014), Southport, UK. Recuperado de http://ewic.bcs.org/content/ConWebDoc/54184

- Kutner, L. y Olson, C. (2008). Grand theft childhood: the surprising truth about violent video games and what parents can do. New York, NY: Simon \& Schuster.

- Kestenbaum, G.I. y Weinstein, L. (1985). Personality, psychopathology and development issues in male adolescent video games use. Journal of the American Academy of Child Psychiatry, 24, pp.329-337.

- Lasswell, H. D. (1948-1985). Estructura y función de la comunicación en la sociedad. En: M. Moragas (Ed.). Sociología de la comunicación de masas. II Estructura, funciones y efectos, pp. 50-68. Barcelona: Gustavo Gili.

- Leal, A. (2002). Narraciones audiovisuales y representaciones infantiles: los roles masculino y femenino. Cultura y Educación, 14(3).

- Levis, D. (1997). Los videojuegos: un fenómeno de masas. Barcelona: Paidós.

- Lezcano, A. (1999). Las miradas sociológicas sobre los procesos de socialización. En: Carli, S. (Comp.). De la Familia a la Escuela. Infancia, socialización y subjetividad, pp. 41-75. Buenos Aires: Santillana.

- Propper, F. (2007). La era de los superniños. Infancia y Dibujos Animados. Buenos Aires: Alfagrama.

- Scott, D. (1995). The effect of video games on feelings of aggression. Journal of Psychology: Interdisciplinary and Applied, 129(2), pp.121-132. doi 10.1080/00223980.1995.9914952

- Shaw, E. F. (1979). Agenda-Setting and Mass Communication Theory. International Communication Gazette, 25, pp.96-10.

- Sherry, J. (2001). The effects of video games on aggression: a meta-analysis. Human Communication Research, 27, pp.409-431. 
Revista de la Escuela de Ciencias de la Educación, año 14, nRo. 13, vol. 1, enero a Junio de 2018. Páginas 149-165. ISSN 1851-6297 (DESDE DICIEMBRE DE 2006 A DICIEMBRE DE 2017). ISSN 2362-3349 (EN LINEA). VIDEOJUEGOS Y VIOLENCIA: Una Revisión de la línea de investigación de los efectos. Santiago García Cernaz.

- Strasburger, V.C. (2007). Go ahead punk, make my day: it's time for pediatricians to take action against media violence. Pediatrics, 119, e1398-e1399. doi 10.1542/ peds.2009-2563

- Tannenbaum, P.H. y Zillmann, D. (1975). Emotional arousal in the facilitation of aggression trough communication. En: Berkowitz, L. (Ed.). Advances in experimental social psychology: Vol. 8, pp. 149-192. New York, NY: Academic Press.

- Tejeiro, R. (1998). La práctica de videojuegos en niños del Campo de Gibraltar. Algeciras: Asociación de Jugadores de Azar en Rehabilitación del Campo de Gibraltar.

- Tejeiro Salguero, R. \& Pelegrina del Río, M. (2008). La psicología de los videojuegos. Un modelo de investigación. Málaga: Aljibe.

- Unsworth, G.; Devilly G.J., y Ward, T. (2007). The effect of playing violent video games on adolescents: should parents be quaking in their boots? Psychology, Crime \& Law, 13(4), 383-394. doi 10.1080/10683160601060655

- Van Schie, E. G. M. y Wiegman, O. (1997). Children and video games: leisure activities, aggression, social interaction, and school performance. Journal of Applied Social Psychology, 27(13), pp.1175-1194.

- Wolf, M. J. P. y Perron, B. (2005). Introducción a la teoría del videojuego. Formats. Revista de comunicación audiovisual, 4, pp.1-27. 